\title{
0.25mm-Thick CCD Packaging for the Dark Energy Survey Camera Array
}

\author{
Greg Derylo ${ }^{\mathrm{a}}, \mathrm{H}$. Thomas Diehl ${ }^{\mathrm{a}}$, Juan Estrada ${ }^{\mathrm{a}}$ \\ ${ }^{a}$ Fermi National Accelerator Laboratory, Box 500, Batavia, IL, USA 60510
}

\begin{abstract}
The Dark Energy Survey Camera focal plane array will consist of $622 \mathrm{k} \mathrm{x} 4 \mathrm{k}$ CCDs with a pixel size of 15 microns and a silicon thickness of 250 microns for use at wavelengths between 400 and $1000 \mathrm{~nm}$. Bare CCD die will be received from the Lawrence Berkeley National Laboratory (LBNL). At the Fermi National Accelerator Laboratory, the bare die will be packaged into a custom back-side-illuminated module design. Cold probe data from LBNL will be used to select the CCDs to be packaged. The module design utilizes an aluminum nitride readout board and spacer and an Invar foot. A module flatness of 3 microns over small $(1 \mathrm{sqcm})$ areas and less than 10 microns over neighboring areas on a $\mathrm{CCD}$ are required for uniform images over the focal plane. A confocal chromatic inspection system is being developed to precisely measure flatness over a grid up to $300 \times 300 \mathrm{~mm}$. This system will be utilized to inspect not only roomtemperature modules, but also cold individual modules and partial arrays through flat dewar windows.
\end{abstract}

Keywords: CCD, packaging

\section{INTRODUCTION}

The Dark Energy Survey ${ }^{1}$ (DES) proposes to build a new camera with a $2.2 \mathrm{deg}$. diameter field of view for the $4 \mathrm{~m}$ Blanco telescope at the Cerro-Tololo International Observatory. The camera will use fully-depleted high-resistivity $\mathrm{CCDs}^{2}$ developed at LBNL. These $2 \mathrm{k} \mathrm{x} 4 \mathrm{k}$ devices have 15 micron pixels and are 250 microns thick for greatlyimproved efficiency at $800-1100 \mathrm{~nm}$. A focal plane array of 62 CCD modules will be constructed, resulting in a 520 megapixel image. A four-side-buttable, back-side-illuminated module design is undergoing development at the Fermi National Accelerator Laboratory (FNAL). In order to minimize the loss of coverage area within the survey array, a gap of $0.5 \mathrm{~mm}$ is planned between adjacent modules. Factors that influenced the initial package design concept include:

- $\quad$ LBNL 2k x 4k high-resistivity CCDs for use between 400-1000 nm

○ $\quad$ CCD size $=63.4 \mathrm{~mm} \times 33.3 \mathrm{~mm} \times 250$ microns thick

- CCD flatness:

$\circ \quad$ On 1 sqcm scales within a CCD, the mean height variation should be $<3$ microns

- For adjacent $1 \mathrm{sqcm}$ areas within a CCD, the difference in mean heights should be $<10$ microns

- Entire CCD surface within 25 microns of the mean focal plane

- Operation in vacuum at about $150 \mathrm{~K}$

- Installation onto the focal plane support plate without shimming

- Easy to install and replace

Early packaging effort has emphasized construction of simple pictureframe-style modules. Six different pictureframe types have been assembled, allowing testing of three different CCD sizes in either front- or back-side-illuminated configurations. All six pictureframe types are shown in Figure 1. In addition to four $2 \mathrm{k} \mathrm{x} 4 \mathrm{k}$ sensors, each silicon wafer contains a $2 \mathrm{k}$ x $2 \mathrm{k}$ sensor, which is intended for use in separate guide, focus and alignment modules, and four $1 \mathrm{k} \times 512$ sensors, which are not intended for use in the camera but which are being utilized during development testing. Three different pictureframe readout card types were fabricated, with window opening sizes and trace layouts corresponding to the three different sensor sizes*. The module's readout connectors are assembled onto the board from different sides

\footnotetext{
* The two smaller sizes were adapted from the $2 \mathrm{k}$ x $4 \mathrm{k}$ pictureframe board, which was designed by LBNL for the SNAP program.
} 
depending on its intended front- or back-side-illuminated use. The CCDs are held in place in the window opening by an aluminum nitride (AIN) ceramic support piece that is epoxied to the pictureframe board. A single AlN design accommodates all front-side-illuminated devices and two additional AlN designs accommodate the three back-sideilluminated pictureframe designs. The back-side-illuminated AlN designs do not fully cover the sensor surface, allowing access for wirebonding between the pads on the CCD and the traces on the pictureframe board. Aluminum nitride was selected due to its similar thermal expansion characteristics to silicon and for its high thermal conductivity. As of April 2006, a total of almost 70 pictureframe modules have been constructed. These devices have been extremely useful for gaining experience with CCD packaging and for development of the FNAL CCD testing facility ${ }^{3}$.

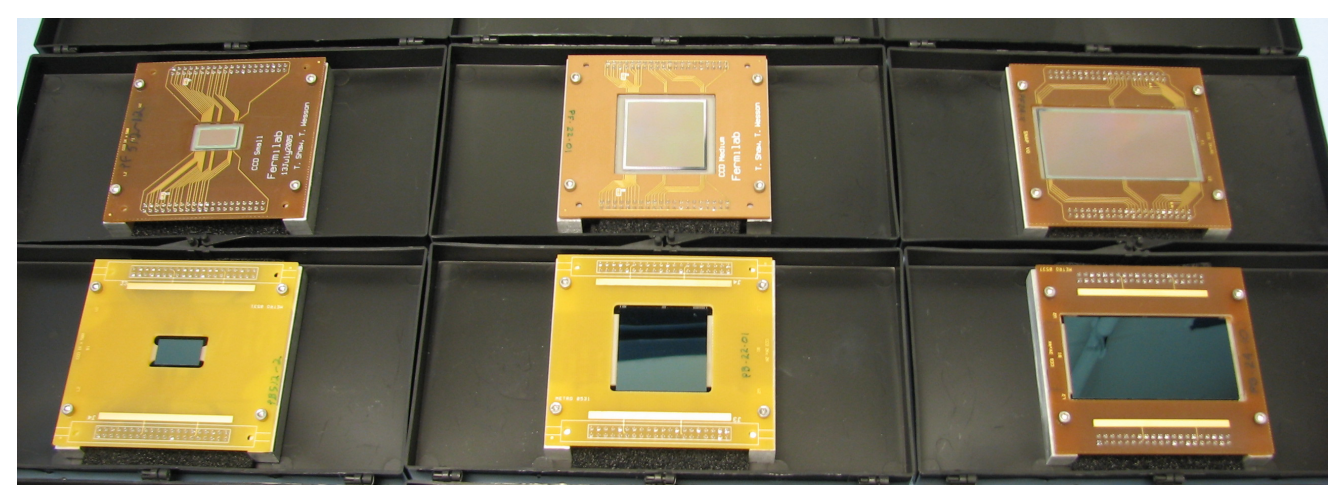

Figure 1. Six different pictureframe package types are pictured. The upper row is front-sideilluminated; the bottom row is back-side-illuminated.

The 4-side-buttable module design being developed uses these $2 \mathrm{k} x$ 4k CCDs and is discussed below. The guide, focus and alignment modules that will use the $2 \mathrm{k}$ x $2 \mathrm{k}$ CCDs have not yet been designed.

\section{MODULE DESIGN}

The initial version of the 4-side-buttable module developed for DES is strongly influenced by previous work ${ }^{4,5}$ with these thick devices. This module is comprised of four main parts, as shown in Figure 2. The CCD itself is oriented with its back side, which has an anti-reflective coating, towards the incoming light. This face must be flat and must have a uniform height relative to the module's mounting surface.

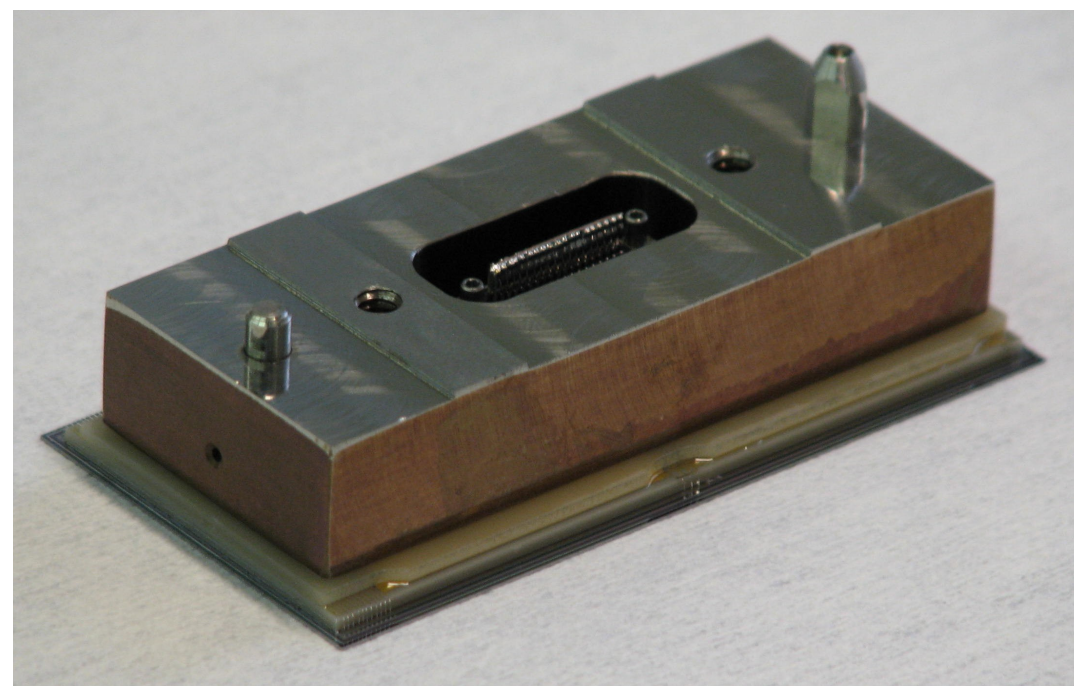

Figure 2. Complete module showing CCD, AlN readout board, AlN spacer, and Invar foot. A temporary shorting plug inserted into the connector is visible. 
The front face of the CCD is epoxied to the $1 \mathrm{~mm}$-thick aluminum nitride readout board. This board has a smaller profile than the silicon so the CCD's wirebond pads, which are located along the long edge of the sensor, are exposed. Aluminum wirebonds are used to connect the pads on the exposed face of the board to the pads on the CCD. A single layer of gold circuitry on the aluminum nitride board connects the wirebond pads along the perimeter to a connector located in the middle of the board. In order to utilize a single layer of circuitry, a few traces had to be routed outboard of the bondpads, between the pads and the board edge. The 37-pin Nanonics connector selected for this board has a mating half that is engaged by tightening two mating screws, and turning these screws in the opposite direction jacks the two connector halves apart, thus minimizing the connection and disconnection forces. Four of the connector's pins are used for readout of a surface-mount RTD temperature sensor, which is mounted to the AlN near the base of the connector.

On top of the readout board is an AlN spacer. This $1 \mathrm{~mm}$-thick piece has a profile nearly the same as the readout board, but it is cut away in some regions to reveal the readout board wirebond pads and has a hole in its center to provide clearance for the connector and the RTD. The readout board and spacer subassembly is shown in Figure 3. The spacer provides a barrier between the exposed gold traces on the readout board and the metal mounting foot. It also increases the overall stiffness of the readout board before the mounting foot is attached, thus helping to maintain module flatness.

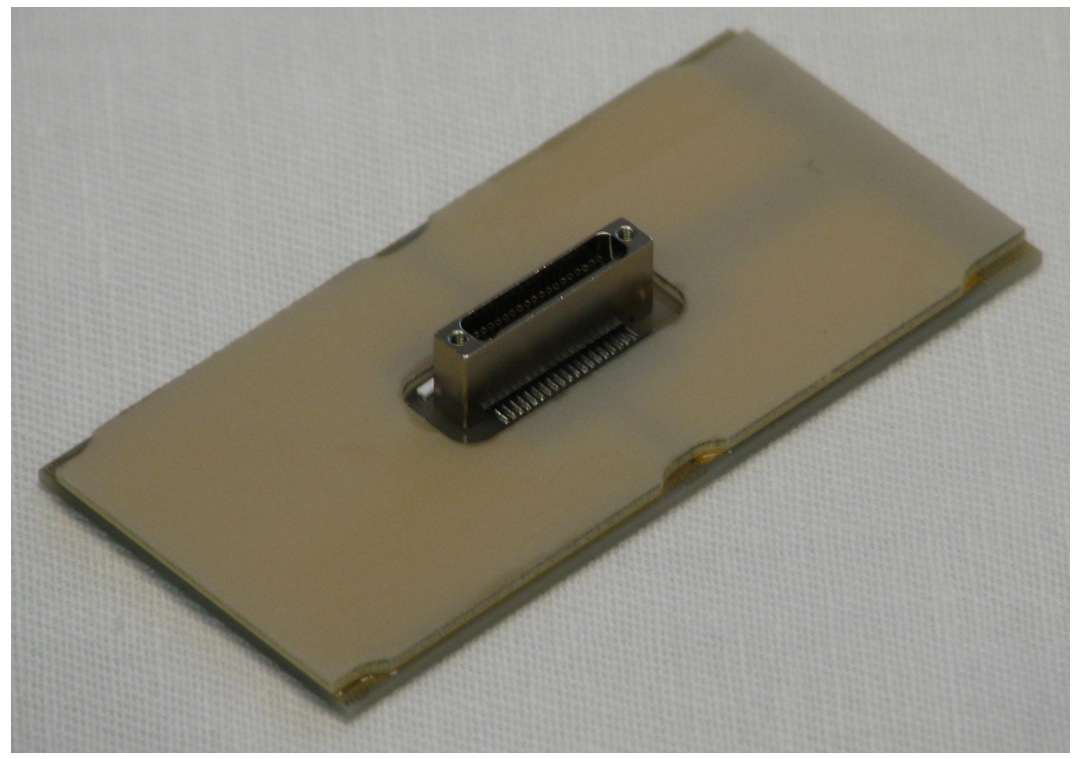

Figure 3. Aluminum nitride readout board and spacer showing the bondpads along the long edges, the 37-pin Nanonics connector, and the white surface-mount RTD.

An Invar foot is mounted onto the AlN spacer. This provides an interface between the focal plane array support plate and the module. Invar 36 was selected due to its good coefficient of thermal expansion characteristics in relation to the silicon and AlN, thus minimizing thermal deformations upon cooling to cryogenic temperatures. The design has a footprint slightly smaller than that of the AlN spacer and has an opening in its middle to allow access to the connector on the readout board. The rear face of the foot has two alignment pins that are used to engage the array support plate. Since the basic module layout is symmetrical, use of alignment pins with different diameters ensures that the modules get installed in the correct orientation. The length of these pins is sufficient to prevent interference between adjacent modules in the array during installation. The rear face of the foot also has two slightly-raised sections that are the primary contacts with the focal plane array support plate. This limits the area over which tight flatness tolerances must be maintained, allowing the rest of this face to be less precise. In the middle of each of these regions is a threaded hole used to fasten the module in place. During module installation, long loading rods are fastened into each of these holes 
and are used as guided installation handles during array assembly. One rod is then locked in place while the other is replaced with a fastener and then the remaining rod is removed and replaced with a fastener.

Each of the three epoxy joints within this assembly are made using Epotek 301-2 epoxy. The ability of this lowviscosity product to fill gaps between parallel plates by capillary action is relied upon during module construction, as discussed in Section 3.

The estimated temperature distribution within a module is shown in Figure 4, which indicates that the gradient through the package is about $3 \mathrm{~K}$. More than half of the gradient is within the foot, which is expected due to the low thermal conductivity of Invar. But the epoxy joints also provide significant thermal resistance, as indicated by the clear differences in the uniform temperatures in the silicon, two aluminum nitride layers, and the bulk of the foot. Due to the differences in module component material properties and the large difference between the construction and operating temperatures, thermal distortion effects must be understood to ensure that the module remains flat. The predicted thermally-induced deformation is also shown in Figure 4. The maximum warpage across the CCD face was estimated to be about 2 microns at $-100^{\circ} \mathrm{C}$ and about 5 microns at $-125^{\circ} \mathrm{C}$.
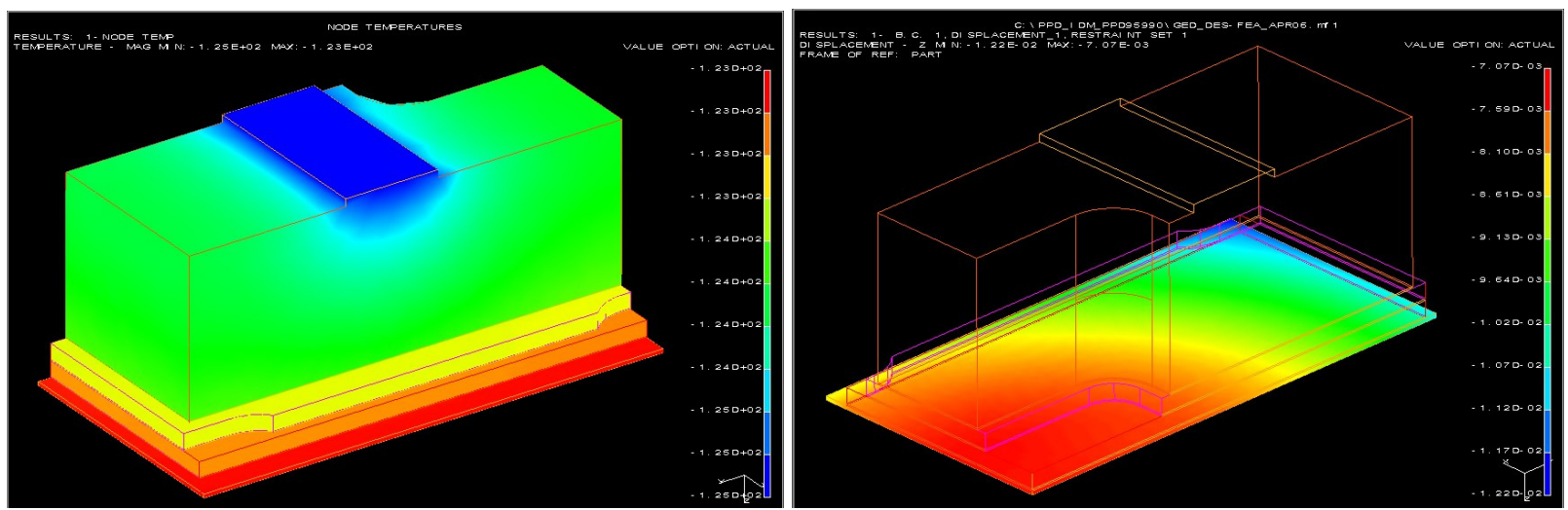

Figure 4. Quarter-section model showing the estimated temperature distribution and thermally-induced distortion when cooled to $-125^{\circ} \mathrm{C}(148 \mathrm{~K})$. The results indicate a $3^{\circ} \mathrm{C}$ gradient through the module and a 5 micron CCD thermal deformation.

\section{MODULE ASSEMBLY}

Prior to shipment of the CCDs from LBNL, each of the $2 \mathrm{kx} 4 \mathrm{k}$ sensors is cold probed to evaluate its potential performance. This information is used to choose the role of each CCD, with lower-quality devices having been selected for initial packaging research and development. Upon arrival of CCDs at FNAL, basic information on each sensor is loaded into a database that is used to help track the use of each device. Paper handling forms are assigned for each sensor serial number and accompany each device at all times so all handling operations can be noted.

After removing sensors from the wafer tape, the front side of each device is visually scanned for irregularities using a small coordinate measuring machine outfitted with a video microscope. Features larger than a pixel are noted and their position is measured for future reference if needed during device testing.

The first step in the construction of the module is the gluing of the readout board and spacer subassembly. After the connector and the RTD have been soldered to the aluminum nitride readout board, the AlN spacer is epoxied to its top surface. A simple jig is used to ensure that the edges are properly located such that the spacer does not overhang the edge of the readout board and adequate clearance is left to the wirebond pads. The gluing technique used to join these parts is similar to that used in previous packaging work with these sensors ${ }^{5}$. Small squares of double-sided tape, approximately $1.5 \mathrm{~mm}$ square and 100 microns thick, are placed in each corner and maintain the relative position of the two aluminum nitride parts with a small gap between them. The low-viscosity Epotek 301-2 epoxy is then fed into the joint, and capillary forces draw the adhesive into the gap. Because of the slightly translucent nature of the AlN, the 
progress of this migration can be directly observed through the spacer material. Several of these subassemblies can be glued in a single sitting, gaining efficiency in the amount of time spent per module.

Once the aluminum nitride readout subassembly has been completed, it can be glued to a CCD. The sensor is placed on a fixture, with its back (light-collecting) side facing downwards and resting on a cleanroom wipe. The edges of the CCD are registered against pins in the fixture to set its position and vacuum is applied to small grooves in the fixture's surface to maintain the sensor's position. An additional set of pins is inserted into the fixture and used to guide the edges of the aluminum nitride readout board subassembly, which is lowered onto the CCD. This board has small squares of double-sided tape that are applied to its bottom face, which is the side that will be epoxied to the CCD surface. Six pieces of tape are used; one in each corner and one each in the middle of the long edges. These last two were utilized to help maintain the 100 micron gap more uniformly between the CCD and the aluminum nitride as the unsupported silicon was found to have a natural bow about equivalent to the tape thickness, resulting in a restriction in the gap between parts. The Epotek 301-2 epoxy is then fed into this joint at a point away from the wirebond pads. Unlike the previous gluing step, the migration of this epoxy through the gap cannot be directly observed. Rather, the generation of a slight fillet around the perimeter of the readout board is used as an indicator of the epoxy's progress.

Allowing the epoxy to cure in this form would not result in a flat device since the CCD is not well constrained and its flatness is therefore still influenced by its natural, free-condition shape. Therefore the subassembly is transferred to a precision-ground vacuum plate where the CCD face is held flat during the two day room temperature cure. This plate, from Tru-Stone Technologies, has a porous ceramic surface to which vacuum can be applied and is ground flat to 1 micron over $150 \mathrm{~mm}$. Unused areas of the plate are masked off to minimize vacuum leakage and maximize the holding force on the CCD. This technique has been successfully used in previous packaging work ${ }^{5}$ with these devices.

After curing, the module is removed from the vacuum plate, placed on a covered carrier plate, and transferred to wirebonding. The carrier plate supports the device during bonding so no additional handling is required. It contains a vacuum channel that connects via a small through hole to a vacuum channel in an adapter plate on the wirebonding machine, allowing the vacuum to both hold the carrier plate in place and firmly hold the module during bonding. Forty aluminum wedge bonds connect the AlN board to the CCD.

Once bonded, another fixture is used to attach the Invar foot subassembly, which already has its alignment pins installed. This fixture is comprised of a base plate, which supports the silicon, a top plate, to which the foot is attached, and a set of spacers, which keep the fixture top and bottom plates apart to regulate the overall package height. These parts are shown in Figure 5. The surfaces of the top and bottom plates are ground flat to within a few microns. The bottom plate has a set of pins that locate the edge of the silicon, which is held in place with vacuum, and another set of pins that control the position of the top plate. The top plate has holes that engage the alignment pins on the foot, which is held firmly in place with screws inserted into the threaded holes used for attachment of the foot to the focal plane array support plate. Three spacers separate the top and bottom plates, which are clamped together using threaded studs that pass through the center of each spacer. The spacers are each ground to the nominal overall module height, and when assembled the fixture leaves a gap of about 100 microns between the Invar foot and the AlN spacer. The ability of the Epotek 301-2 epoxy to be drawn into gaps by capillary action is again utilized here to join the upper and lower halves of the module. By accurately constraining the positions of these upper and lower subassemblies and filling the remaining gap with adhesive, it is intended to achieve modules which have a uniform thickness part-to-part. 


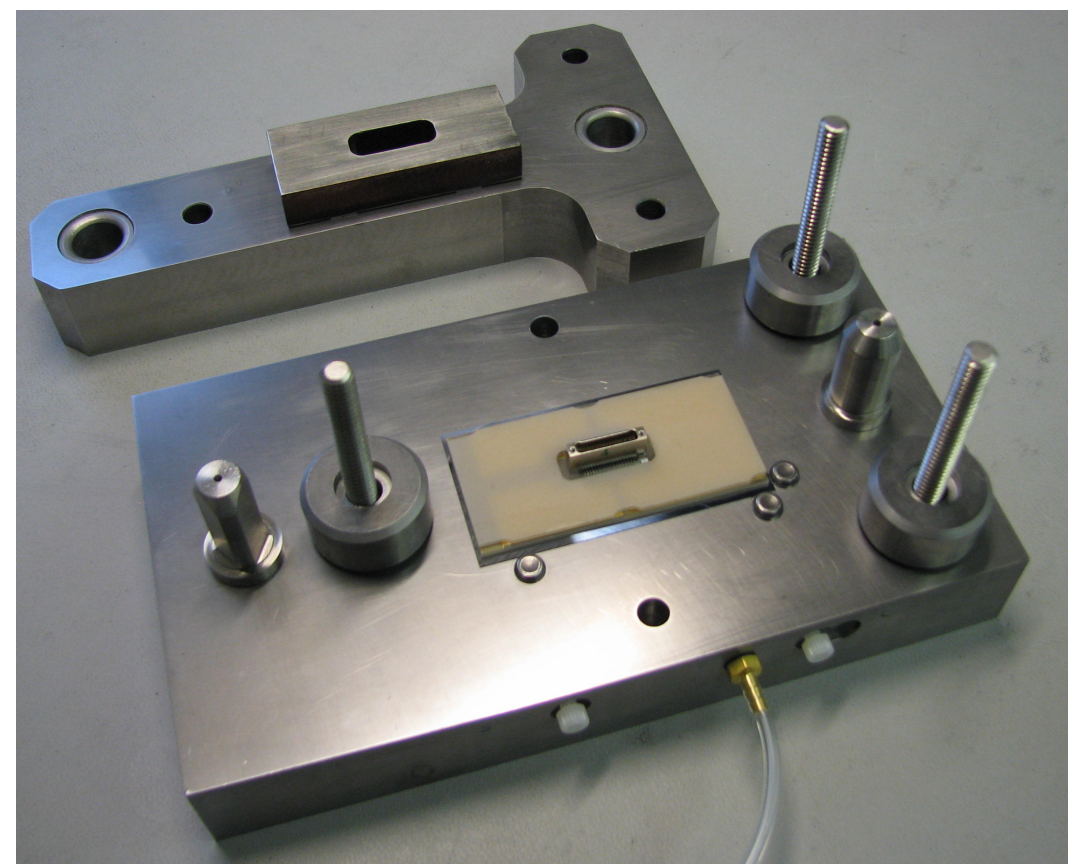

Figure 5. The foot gluing fixture shown with the upper and lower halves of a module prior to gluing.

Once complete, the module is removed from the fixture and transferred into a storage box (Figure 6). In order to minimize device handling, the storage box design allows testing without removal of the CCD. The box interfaces directly with the testing dewar and allows access on the underside to the module's connector. After cabling the module and fastening the box to the test dewar coldfinger, the box cover is removed and the vessel is sealed.
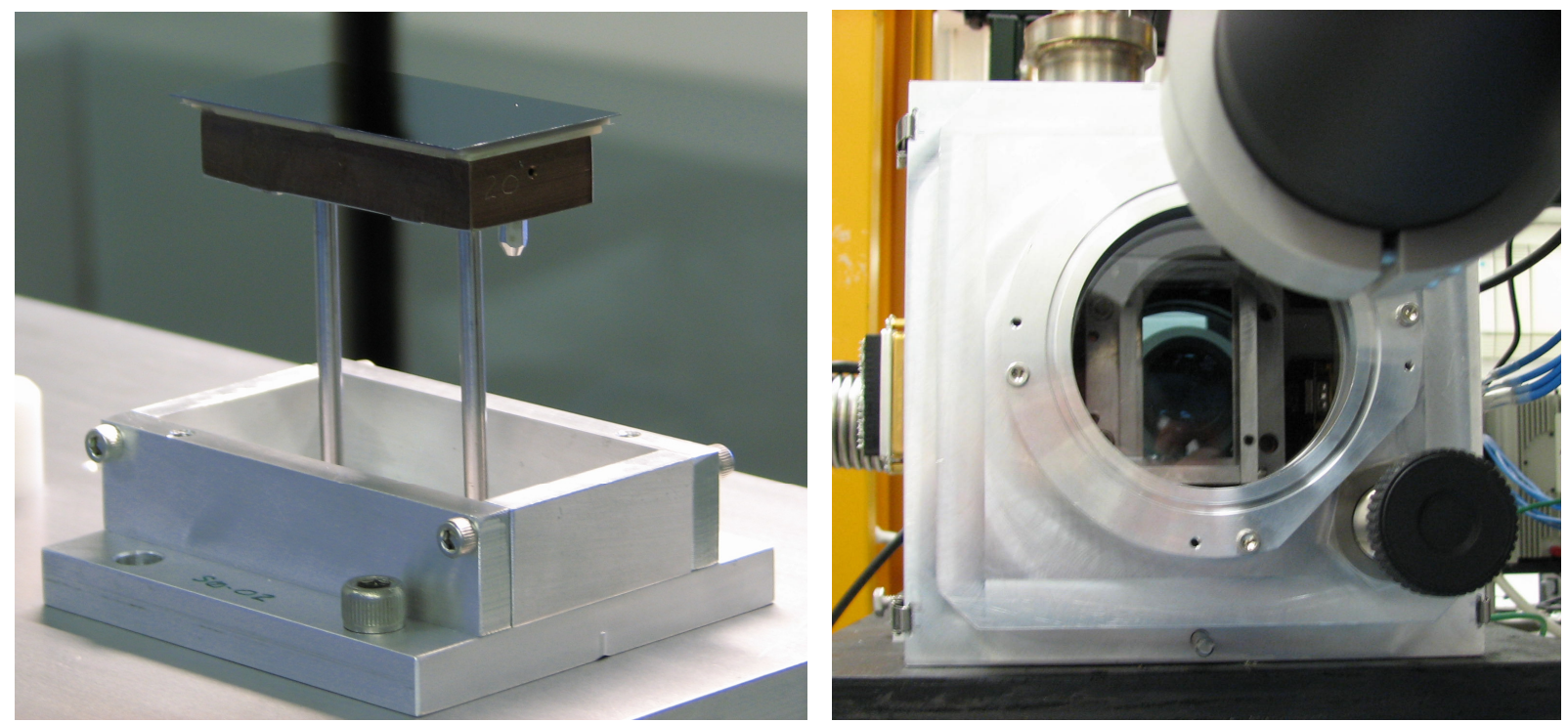

Figure 6. Module storage box, shown while loading and after installation into the test stand. 


\section{MODULE PERFORMANCE}

Two modules have been constructed using the methods described in Section 3. After completing the foot gluing operation, the module thickness is measured optically at several points. The top plate of the foot gluing fixture, while it still has the completed module fastened to its flat ground surface, is placed on a small coordinate measuring machine equipped with a microscope camera. Using camera focus to inspect heights, a grid of 15 elevation points is measured on the exposed surface of the CCD relative to the flat fixture plate to which the module is mounted. The repeatability of the camera autofocus when measuring the CCD is \pm 1 micron. Figure 7 shows the measurement results for the two modules relative to the nominal design thickness. The tilt present in the first module was improved for the second module, for which increased torque was applied on the studs clamping the foot gluing fixture parts together. Performing this step with better uniformity is expected to yield more consistent results for the next set of devices.
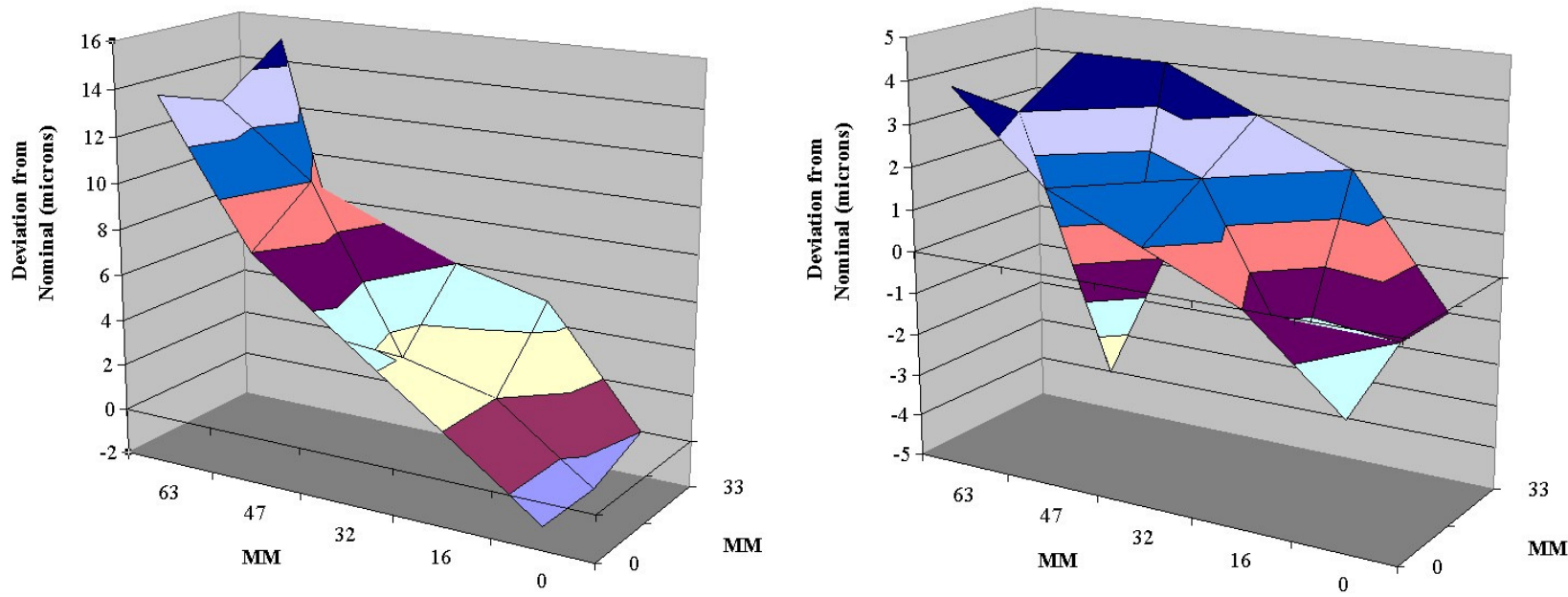

Figure 7. Thickness deviation from nominal for two modules.

Although the system used to measure package thickness might be used to perform a more detailed inspection of CCD flatness using a finer grid pattern, such an approach is not feasible for inspection of a module when cooled to its operating temperature. A system for performing this inspection would have to have a greater stand-off distance, allowing space for the vacuum vessel hardware required for operating under these conditions, including performing this inspection through the vessel window. Ideally, such a system would also have to have the ability to travel over a large enough surface area such that the completed camera focal plane, or at least a significantly large portion of it, could be inspected in both warm and cold conditions. A flatness scanning system is being developed at FNAL using a confocal chromatic displacement measurement system from Micro-Epsilon Corp. This device takes light from a halogen bulb and directs it such that light of different frequencies is focused at different distances from the instrument head. The frequency of the light reflected back from the focus spot is sensed by the system and is an indicator of the illuminated surface's distance from the instrument head. The advertised accuracy of this device is approximately 7 microns linearity over a $24 \mathrm{~mm}$ range with a maximum working distance of almost $250 \mathrm{~mm}$. The positioning system used to guide the scanning head must have exceptional straightness in order to utilize the accuracy of the instrument, for variations in stage straightness will directly impact measurement accuracy. A set of motorized stages from Physik Instrumente with a straightness of 1 micron per $100 \mathrm{~mm}$ of travel was therefore selected. The $300 \times 300 \mathrm{~mm}$ travel range of the system is not large enough to measure the entire surface of the completed camera array, but it will allow coverage of a significant portion of it such that the flatness of the entire array can be mapped from a small number of overlapping measurement fields. The scanning system is shown in Figure 8. 


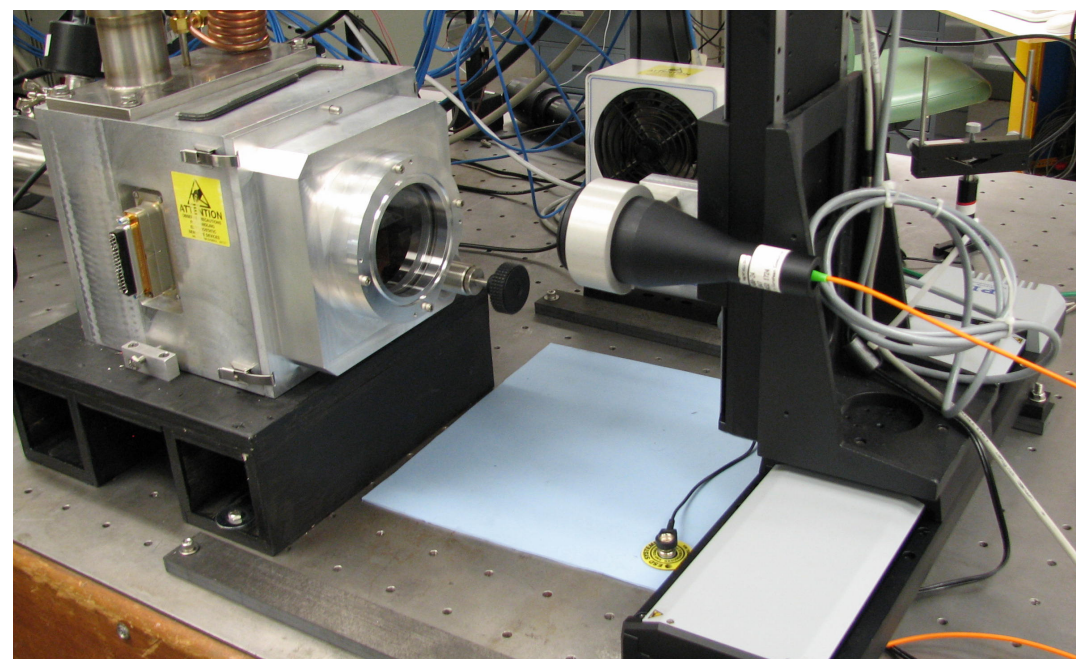

Figure 8. Confocal-chromatic CCD flatness scanning system positioned in from of one of the test stations.

The scanner and translation stages are controlled with a LabView program designed to survey a grid of points on the CCD surface. The grid spacing is an input parameter and we typically use a 500 micron step. Each data point is actually the average determined from 20 consecutive readings taken at that position and the standard deviation of the measurements is typically 4 microns. A full scan of the surface, with about 7800 grid points, takes about 90 minutes.

The accuracy of the system has been tested using a device with two small CCDs separated by a $4 \mathrm{~mm}$ height difference. Even with the antireflective coating, and measuring through the vacuum vessel window, we measure the height difference to better than $2.5 \%$. The measurements are repeatable to within the accuracy of the system, though we have to control systematics including vibration and thermal expansion of the optical table.

Flatness measurements of the single module that has been scanned to date (we have scanned several test surfaces) are shown in Figure 9 and correspond to the module whose thickness measurement is shown in the right-hand plot of Figure 7. The leftmost plot shows the flatness measured at $294 \mathrm{~K}$. The surface of the CCD has been divided into 325 subsurfaces and the measurements in each subsurface have been averaged together to provide an overall precision of approximately 0.4 microns in each subsurface. These measurements indicate a larger flatness variation, 15 microns peak-to-peak, than was found in the thickness measurement. The RMS flatness variation is approximately 2.7 microns. The reason for the difference between the flatness and the thickness measurements is not yet understood, but there are obvious differences in the equipment and setup of the two measurements. The middle plot shows the flatness scan results at $152 \mathrm{~K}$. The peak-to-peak flatness variation is about 20 microns and the RMS variation is 2.8 microns. A significant portion of this variation was measured at the extreme corners of the device. The right-hand plot shows the difference between the two cases. Inspection indicates the general trend for the CCD is to bend so that the short edges are lower than the corresponding slice across the middle. Indeed, comparing the average height $2.5 \mathrm{~mm}$ from either short edge with the average height across the middle indicates the bend is 8.4 plus or minus 0.9 microns. The analysis shown in Figure 4 had predicted a deflection of just over 5 microns at this temperature. 

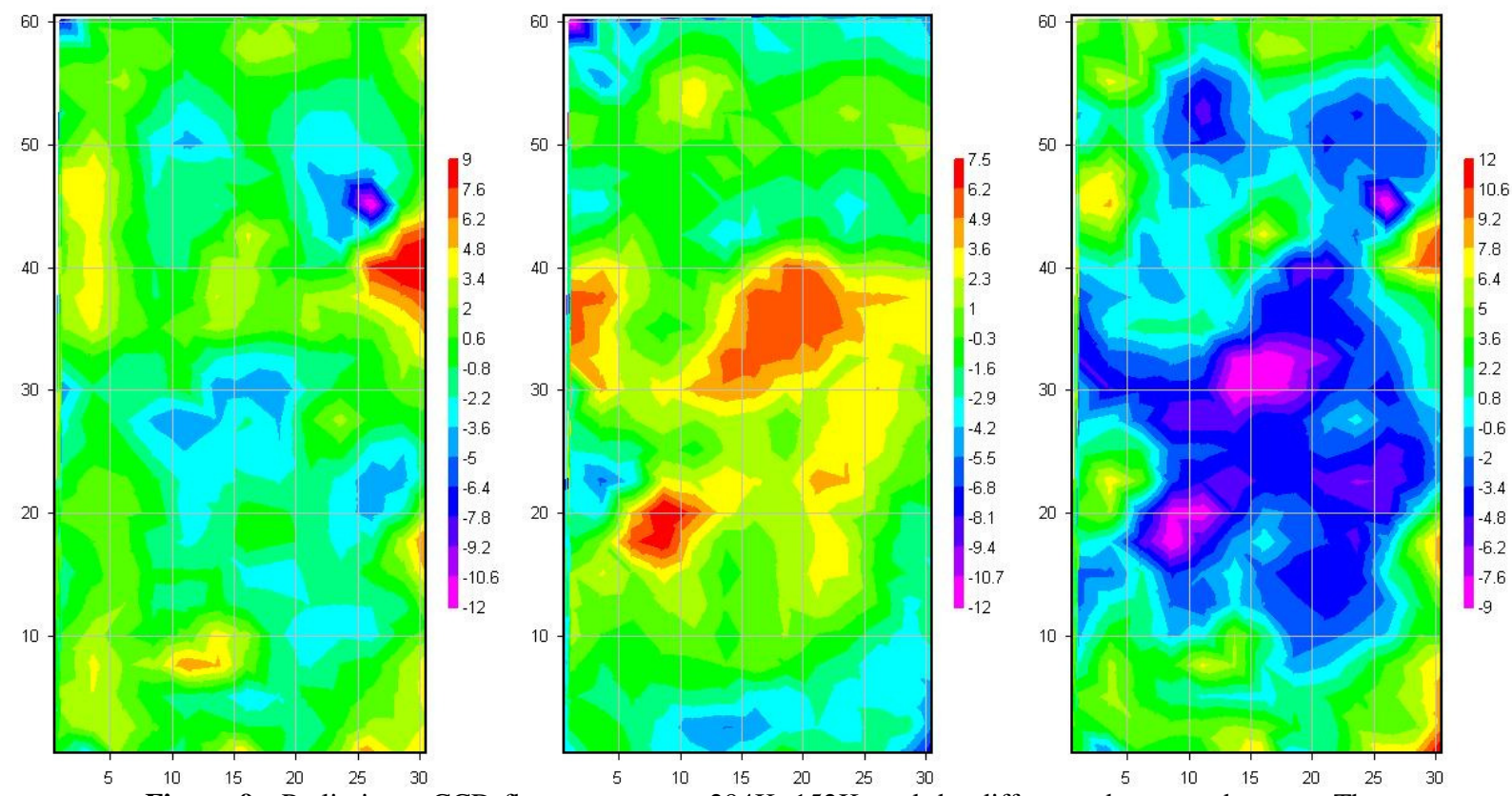

Figure 9. Preliminary CCD flatness scans at $294 \mathrm{~K}, 152 \mathrm{~K}$, and the difference between the two. The positive direction is away from the scanning instrument, representing low points on the CCD.

A small area flatness scan on a finer step size was performed in the region of one of the small double-sided tape pieces used to tack the CCD to the AlN readout board during assembly. These measurements indicate that the surface of the $\mathrm{CCD}$ is distorted, at $152 \mathrm{~K}$, by the presence of the tape; indeed, the effect is just visible to the eye as tiny irregularities in the locations of the tape. Measurements of the amplitude of the bump are still in progress.

The tape pieces used to tack the CCD in place during assembly were also observed in CCD images, as shown in Figure 10. Images obtained over 400-1000 nm indicate that these features are present over the full frequency range. Although these features might be cosmetically removed when corrected with flat-field images, the concern over geometric distortion is leading towards identifying an alternative to this assembly technique.

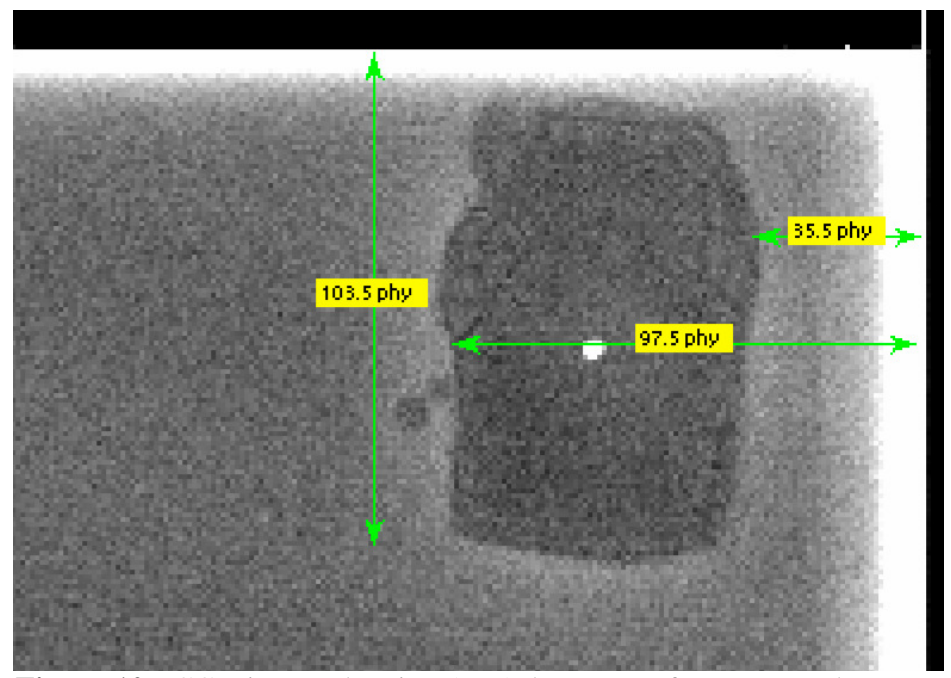

Figure 10. CCD image showing 1 x $1.5 \mathrm{~mm}$ tape feature near the sensor corner. The feature is visible at all frequencies. 


\section{CONCLUSION}

The development effort of the DES CCD packaging continues. The experience gained on working with these devices has been very useful in exploring aspects of module design, in procurement and fabrication of module parts, in handling and working with CCDs, and in inspection and testing of completed devices.

Although a previously-developed successful technique ${ }^{5}$ for the production of flat devices has been utilized here, the fine-grid flatness inspections being performed with the confocal chromatic system indicates that the measurements of the single device scanned to date does not satisfy the requirements of the project. We are investigating our packaging and measurement system issues so flat modules can be successfully produced and inspected.

DES project plans include fabrication of additional modules with existing parts of the current version in order to assemble a test array of six devices. Revisions to the module design will then be made based on our experience packaging this initial package version. Currently-intended design improvements include:

- Development of an alternative CCD-to-AlN gluing process to eliminate the double-sided tape used to tack these components together.

- Attempt to reduce the overall module height so wirebonding can be performed on a completed device rather than at an intermediate assembly stage. This would require moving alignment pins from the module's foot to the focal plane support plate.

Design improvements made to the $2 \mathrm{k}$ x $4 \mathrm{k}$ module configuration will also be incorporated into the design of the guide, focus and alignment packages that will utilize the $2 \mathrm{k}$ x $2 \mathrm{k}$ CCDs.

\section{ACKNOWLEDGEMENTS}

We would like to extend our gratitude to Jim Fast, who designed the flatness scanning system, and Selcuk Cihangir, who assisted with its programming. We would also like to thank Jim Wish for his work helping to package the CCDs. The Fermi National Accelerator Laboratory is operated by Universities Research Association Inc. under Contract No. DE-AC02-76CH03000 with the United States Department of Energy.

\section{REFERENCES}

1. B. Flaugher for the DES Collaboration, "The Dark Energy Survey Instrument Design," these proceedings.

2. S.E. Holland, et al., "Fully Depleted, Back-Illuminated Charge-Coupled Devices Fabricated on High-Resistivity Silicon," IEEE Transactions on Electronic Devices 50 (3), 2003.

3. J. Estrada et al., "CCD Testing and Characterization for Dark Energy Survey," these proceedings.

4. H.M. Oluseyi, et al., "LBNL 4-Side Buttable CCD Package Development," Proceedings of SPIE 5301, 2004.

5. R. J. Stover, et al., "Packaging Design for Lawrence Berkeley National Laboratory High Resistivity CCDs," Proceedings of SPIE 5499, 58, 2004. 